\title{
A Fuzzy Logic Model for the Container Stacking Problem at Container Terminals
}

\author{
Jana Ries ${ }^{1}$, Rosa G. González-Ramírez ${ }^{2}$, and Pablo Miranda ${ }^{2}$ \\ ${ }^{1}$ Portsmouth Business School, University of Portsmouth, Portland Street, \\ Portsmouth, PO1 3DE, UK \\ jana.ries@port.ac.uk \\ ${ }^{2}$ Industrial Engineering School, Pontificia Universidad Católica de Valparaíso, \\ Av. Brasil 2241, Valparaíso, Chile \\ \{rosa.gonzalez, pablo.miranda\}@ucv.cl
}

\begin{abstract}
We address the problem of storage space allocation in a sea port terminal. The problem consists of assigning a block space in the yard of a container terminal to every incoming container while ensuring operational efficiency. The proposed framework uses a 2-stage framework in combination with a fuzzy logic rule-based strategy. The concept is motivated by the problem faced by container terminals in Chile and the aim is to provide real-time decision support to deal with a high degree of uncertainty in the arrival of containers at the yard. In addition, the framework provides a more flexible design to include a set of different criteria as well as different infrastructures and layouts of container ports. Numerical results are presented, comparing the results of the fuzzy framework with respect to algorithms proposed in the literature, considering different scenarios.
\end{abstract}

Keywords: Storage space allocation, container terminal operations, fuzzy logic, online decision support.

\section{Introduction}

Container terminals play a critical role in supply chain management as intermodal interfaces [31], with competition between container terminals increasingly demanding more efficient cargo handling. Due to governmental globalization and trade facilitation efforts to enhance international trade, container terminals face an increasing number of containers to be handled. Moreover, different strategies employed by shipping companies such as the redesign of routes, the generation of alliances and the introduction of bigger sized ships to achieve economies of scale are putting pressure on the ports to be able to efficiently respond to the current demand of increasing container traffic.

Container terminals can be divided into three main areas: quay, yard and gate. Operations are divided into seaside (quay) and landside (gate), with the yard acting as a buffer area that services both the quay and the gate. Seaside operations consist of the assignment of berths to ships as well as the quay cranes that will service each ship.

R. G. Gonzáez-Ramírez et al. (Eds.): ICCL 2014, LNCS 8760, pp. 93-111, 2014.

(C) Springer International Publishing Switzerland 2014 
Moreover, scheduling of the jobs of each quay crane should be defined as well as the stowage plan for the loading and unloading of containers at the ship. In practice, these decisions are interrelated and linked to yard and gate operations at the port. Yard management on the other hand, consists of the assignment and scheduling of yard equipment (i.e., yard cranes/internal trucks) both for seaside and landside operations, as well as the storage space allocation and container stacking policies. More details can be found in $[5,36,37]$.

In this paper, the container stacking problem is addressed which searches for an order or pattern in which containers shall be moved into a yard for temporary storage. Containers are stacked along bays and rows, which determine the length and width of a block, respectively. Tiers of the block determine the height (number of levels) of each block. A stacking position, corresponding to a bay, row and tier, has to be selected for each container that needs to be placed into the yard. A good storage space assignment reduces the storage yard operations cycle time (i.e., the time to store, retrieve, and reshuffle or relocate) [8].

Maximizing storage space utilization is of particular interest for those terminals with space limitations, as it is the case for several ports in Latin America. Minimizing the time required for internal trucks to transfer containers between the yard and the quay is also an objective to consider, given that this enhances service levels as it reduces dwell times [9]. Various problem modeling approaches exist in the literature considering individual or a combination of multiple objectives.

Several container stacking strategies have been proposed in the literature. Two categories of stacking strategies are distinguished by [10]: category stacking (where containers of the same category are stacked on top of each other) and residence time strategy (where a container is stacked on others if the departure time is earlier than that of all containers below). On the other hand, Steenken et al. [36] distinguish storage planning and scattered stacking. In storage planning, space in specific areas of the stack is reserved before the ship's arrival. In scattered stacking, yard areas are not assigned to a ship's arrival but to a berthing place. The stacking position is then determined in real-time and containers are stochastically distributed over the area. Scattered stacking results in higher yard utilization and a significant reduction in the number of relocations or reshuffles. Relocations of containers occur when a container that should be retrieved is not directly accessible to the yard crane and hence, additional moves of other containers are required to retrieve the target container. These inefficient movements result in additional costs and service times to the terminal.

A selection of decision makers at a Chilean port who motivated this study outline the most important criterion to be the minimization of relocations. Other measures to assess yard efficiency include the congestion within the yard infrastructure such as waiting times due to a high level of utilization as well as the distance travelled by internal trucks.

The presented framework addresses the challenge of online decision support by means of multi-criteria decision analysis using a fuzzy logic framework which is applied to the storage space allocation moves and the relocation moves. While static optimization modeling uses a rolling time horizon to determine a yard plan, real-time decision making allows immediate consideration of unexpected events including 
delays in container (or ship) arrival in the yard. The inclusion of fuzzy logic ensures flexibility with regards to criteria under consideration. The literature consists of several studies on the basis of evaluating the stacking position considering a set of criteria, i.e. $[1,9,28,39]$, or a weighted combination of criteria [29], but none of the previous approaches has considered a fuzzy logic model.

The remainder of the manuscript is organized as follows. Section 2 presents a literature review of yard management problems. Section 3 presents the problem description and the modeling approach, and Section 4 presents the results of a numerical application. Conclusions and managerial insights as well as recommendations for future research are given in Section 5.

\section{$2 \quad$ Literature Review}

Several authors have studied problems related to logistics operations within the terminal, at strategic, tactical, and operational levels. A description and classification of the main logistics processes and operations in container terminals and a review of the methods that have been proposed to optimize the main operations in the terminal are presented in [35, 36]. In [2] a survey of the different problems and modeling approaches for ship planning problems is presented, analyzing also the different solution methodologies that have been proposed in the literature, providing a classification scheme. In [5] an in-depth analysis of storage space allocation problems at container terminals is presented, and a classification scheme of the yard operations is used to classify the literature that is found between 2004 and 2012.

Strategic decisions at the yard of container terminals are related to layout design and the acquisition of handling equipment [23, 27]. For tactical and operational level decisions, several contributions can be found in the literature concerning container handling policies and real-time decisions. Some of the problems addressed are related to the scheduling of yard cranes, routing of internal vehicles and cranes, allocating storage spaces to containers, and stacking policies. For scheduling and routing of yard equipment the reader may refer to the work by $[19,22,26]$.

One of the earliest contributions that address the storage space allocation problem is provided by [18] who consider the storage space allocation problem for import containers so as to minimize the number of re-handles. In [21] dynamic programming is used to determine the storage locations of export containers using weight groups. In [20] the problem of allocating storage space to export operations is addressed based on a mathematical formulation. The model attempts to minimize the traveling distance between yard and quay movements of containers. Regarding the assignment of space to individual containers or stacking strategies, several authors have been analyzing this problem, either to determine stacking policies or to assigning decisions with the aim to minimize container relocations. The reader may refer to $[8,15,16,33$, $37,38]$.

In [42] the storage space allocation problem (SSAP) is formulated. A two-stage problem formulation is proposed, that aims to balance the workload among blocks and minimizing the total distance traveled. An extension of the SSAP formulation of [42] 
is provided by [1] to consider different types of containers but only for the case of import flows. A comparison of stacking strategies in terms of unproductive movements is presented in [25]. A simulation approach is employed to compare a random and ordered strategy, as well as different layout configurations of the yard. An online space allocation model which considers real-time allocation of containers in the yard is presented in [29], considering an automated container terminal. A detailed explanation of the difference between a conventional and automated container terminal is presented. The work presented herein considers a conventional terminal that is operated with RTGs (Rubber Tyred Gantry cranes).

Online container stacking methods for an automated terminal are proposed by [10], based on a scattered stacking and category stacking strategy. The aim of the heuristic proposed is to search a pile that is not full with containers of the same category and ship to locate a container on top. Two strategies for container stacking are proposed by [3] based on departure times and the trade-off between stacking further away in the terminal versus stacking close to the exit points and accepting more reshuffles. They consider several residence time classes and use that information to limit the number of relocations. A container terminal with high uncertainty with respect to departure times for import containers is considered. They compare a number of stacking rules where trade-offs are considered between further traveling and the possibility of reshuffles. Three stacking strategies are introduced in [32] that take into account the containers' arrival and departure rates and the storage yard characteristics. Results show that the optimal strategy depends on stacking height and the relationship between vessel headway and container dwell time.

In [9] the location assignment problem for outbound containers is addressed. The problem is related to ours, as they consider groups of containers, but based only on weight information while in our case we can consider different criteria. A real time container storage system is presented by [30], and a discrete event simulation model of a vessel-to-vessel transshipment terminal to show the impacts of yard storage allocation on the overall productivity of the container terminal, measured as the gross crane rate (number of lifts per quay crane hour). A decision-tree heuristic is proposed by [13] to minimize the expected number of reshuffles when arriving containers that should be stacked in a block of containers with an arbitrary number of piles. The authors compare shared and dedicated storage policies and results show that sharedstacking outperforms dedicated-stacking.

The main difference with respect to previous works is that we employ a fuzzy logic strategy that has as an advantage that different characteristics can be implemented without increasing the complexity of the procedure.

Integrative approaches of yard storage allocation along other decisions of port operations can be found in the literature. For instance, in [24] the transfer vehicle scheduling problem is integrated with storage space allocation decisions. Another related work is provided by [40] and [43] where the impact of truck announcements on container stacking is evaluated. The blocks relocation problem is addressed by [6] that present a solution procedure based on the Corridor method proposed by [34] as a hybrid metaheuristic. A mathematical model for the blocks relocation problem is presented by [7] and the NP-hardness of the problem is proved. A tree search heuristic 
for the container relocation problem is proposed in [12]. Another related yard management problem is the pre-marshaling where export containers are sorted in a premarshaling process to prepare them to be loaded onto a ship more efficiently. A tree search procedure to solve the problem is presented by [4], a heuristic solution is proposed by [11] and two labelling algorithms for two types of pre-marshaling problems are proposed by [12].

\section{Problem Description}

This work addresses the problem of assigning a position to import and export containers at the yard of a conventional container terminal. We assume that the terminal operates based on a grounded storage, where containers are stored in block stacks by yard cranes. We also consider that there are no transshipment operations. The yard is organized into blocks, which are divided into Bays, Rows and Tiers (BaRoTi coordinate). Bays give the position of the containers relative to the cross section of the yard. Rows give the position of the containers relative to the vertical section of the corresponding bay and tiers give the position related to the horizontal section of the bay. Each coordinate defines the position of a container.

At the yard, blocks are dedicated to a certain type of container (i.e., import/export; dry/reefer/empty; IMO). In general, import and export containers are not assigned to the same blocks, while reefer and IMO containers have special and dedicated storage blocks. Empty containers have also dedicated blocks as this type of container allows being stacked at higher tiers. It is a common practice that containers are segregated into groups with common characteristics.

The type of yard cranes employed influences the storage policies, as well as if it is manually handled or automatized. In Latin America, the degree of automation in container terminals is low so that most terminals are conventionally operated and only new terminals in Brazil and Mexico have a degree of automation. Yard equipment employed are typically RTGs, reach-stackers and top-lifters.

Export operations consider the stacking of arriving containers from the hinterland, which are transported by external trucks. Depending on the storage policies of the terminal, export containers can be directly stored at the export stacking area, or there are some terminals that have a pre-marshaling area where containers are temporarily stored prior to assigning a space at the stacking blocks.

Once external trucks enter the port terminal, they are directed to the stacking area where the container will be stacked by yard cranes, based on resource planning and scheduling policies of the terminal. Export containers are loaded onto the ship based on the stowage plan that has been designed. If export containers are not adequately assigned at the yards, during loading operations there may be relocations of containers, causing additional costs and inefficiencies, as well as increasing ship's time at port.

On the other hand, import operations consist of the dispatching of containers so that they can be transported to the hinterland. At Chilean ports, containers are transported by rail or trucks. Dispatching of containers depends on the policy of the port 
terminal. For instance, dispatching of containers is organized shift by shift based on a random arrival of external trucks that request a specific container. This may generate a significant number of relocations, especially as in most cases, container terminal managers assign space based on experience and quite randomly, aiming to only maximize the utilization of space, due to the high uncertainty and high dwell times of containers.

For the research presented herein, we assume that the yard cranes for all the operations at the terminal are RTGs, which implies an unrestricted access to the containers of the yard. However, the model can be easily extended for other types of yard cranes. The framework outlined in the following sections focuses on Import containers only, while the system can be amended to an Export container space allocation scenario.

\section{$4 \quad$ Fuzzy Logic Framework}

As previously stated, the storage space allocation problem requires consideration of various factors (or criteria). These may vary for different type of containers (e.g. Reefer, IMO, etc.) or different operations (e.g. Import, Export). The following framework uses fuzzy logic as a multi-criteria decision making tool for storage space allocation of Import-Dry containers as an example of how this framework may be implemented to various container groups within a particular yard layout.

The designed system uses a 2-Phase strategy with Phase 1 allowing for the assignment of the block to be considered in the yard and Phase 2 determining the best stack position within the particular block, considering the framework proposed in [29]. Phase 1 and 2 both use a fuzzy logic system to derive the best block and stack position, respectively. Both fuzzy systems aim to determine a 'Value of Goodness' (VoG) for each feasible block (Phase 1) and - once the block has been decided upon - all feasible stacks therein (Phase 2). A container should be assigned to the stack with the highest stack VoG within the block that corresponds to the highest block VoG.

Figure 1 shows an overview of the proposed framework that considers the space allocation for an incoming container to the yard (either from the quay or the gate) and the container retrieval process in which the space allocation of the relocated container is determined (when it is necessary) by the 2-phase fuzzy system previously described.

\subsection{Fuzzy Logic}

Fuzzy Logic is a rule-based approach that has been introduced by [41]. It allows to associate crisp quantitative data of variables (e.g. weight, distance, importance) with linguistic terms (e.g. small, large, high, low, etc.) and to introduce a set of rules in order to determine a value of interest. Hence, a fuzzy system consists of a set of membership functions (see Figure 2), to transform numerical information into linguistic terms, and a rule base to evaluate an output variable of interest given a set of input variables.

Figure 2 shows an example for two membership functions for the input variable distance. The fuzzy concept allows for any incoming value to be assigned to a linguistic 


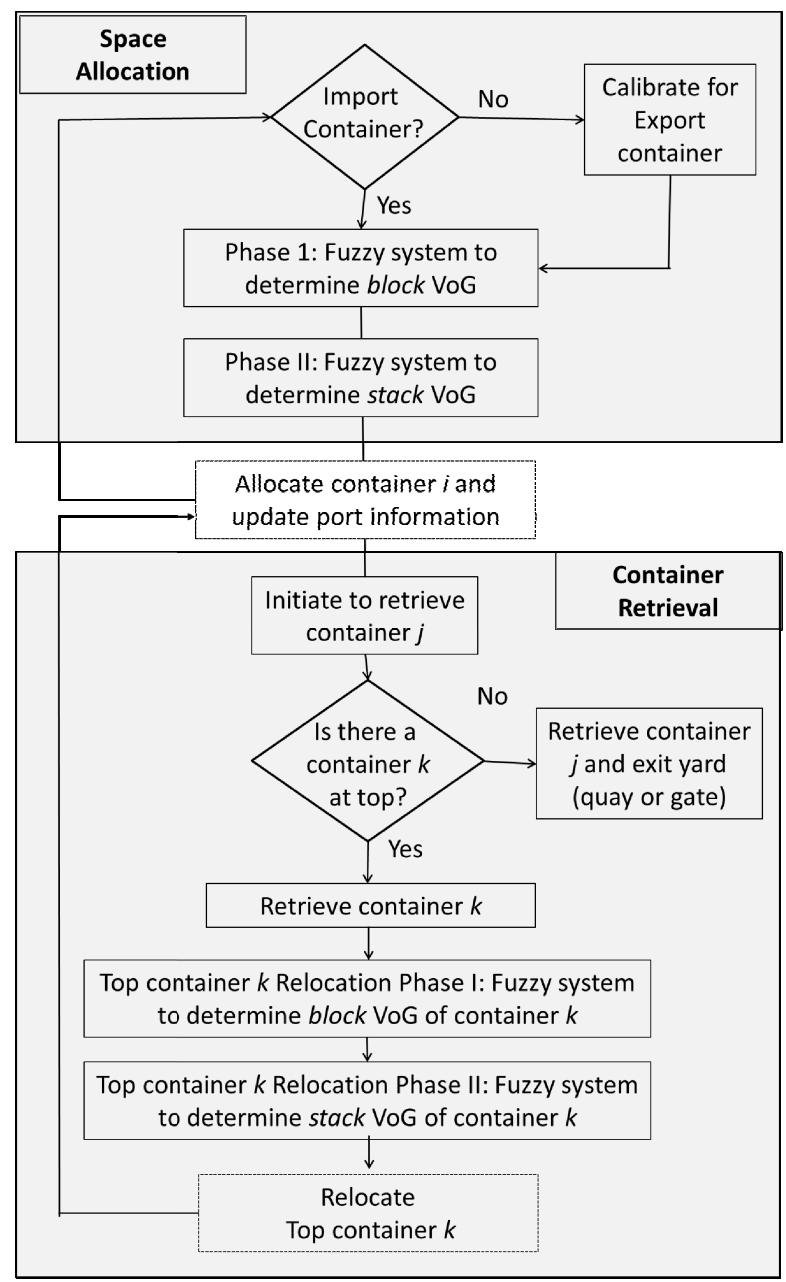

Fig. 1. Fuzzy Logic Framework overview

expression (or membership function) with a certain membership degree. All input variables are assigned to membership functions with a 'degree of membership' between 0 (no membership) and 1 (full membership). Hence, a distance value of e.g. 80 may be considered to belong to the subset large with a small degree of membership but at the same time it is considered to be medium to a certain membership degree. Once the variables have been assigned to all membership subsets, the corresponding rules are activated to determine a combined 'degree of membership' using the AND operator to find the intersection of membership functions of all input variables. Once all relevant rules are applied, the centroid method is used to determining the final output variable value. A fuzzy system uses a rule base that consists of a set of fuzzy rules in the form of IF $\mathrm{a}=$ small and $\mathrm{b}=$ large THEN $\mathrm{c}=$ medium. 


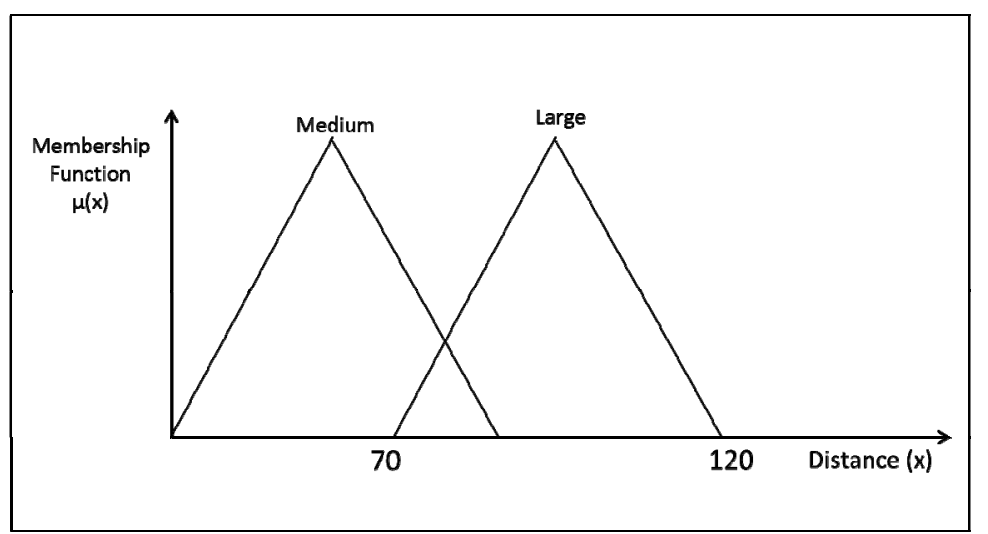

Fig. 2. Illustration of a set of membership functions for the variable Distance

In the framework presented herein, the set of input variables of each fuzzy system consist of container and yard characteristics which are mapped on membership subsets that are labeled by linguistic terms, e.g. 'low', 'high' and 'large'. The corresponding output variables of the system are block VoG in Phase 1 and stack VoG in Phase 2. An example of a set of rules for the presented framework may be as follows (more details are given in the following Sections 4.2 and 4.3).

PHASE I:

-IF (distance to gate $=$ low $)$ THEN $($ block $\mathrm{VoG}=$ medium $)$

-IF (block utilization $=$ low) THEN $($ block $\mathrm{VoG}=$ high $)$

PHASE II:

-IF (current stack height $=$ low $)$ THEN $($ stack $\mathrm{VoG}=$ high $)$

-IF (container weight $=$ low) AND (stack height is high) THEN (stack $\mathrm{VoG}=$ high $)$

A set of decision criteria is considered for the evaluation process, including the height of current stacks, block utilization and container characteristics such as an estimated time for leaving the port or the corresponding dwell time of the container. The overall aim of the proposed allocation system is to increase efficiency by reducing potential relocations and distance traveled of the yard equipment, as both elements directly impact the service time of the vessels which is one of the main performance metrics of the port. Relocations are mainly caused by combining the storage of different containers with different characteristics and different dwell times in the same or close location that when retrieved, cause interference to each other. One strategy may be to allocate containers of the same characteristics (with potential similar dwell times) in one place. However, this will lead to congestion on the paths of internal trucks that transport containers (quay-block or gate-block) as well as the development of queues within the particular chosen area within the yard. Other potential performance measures are therefore waiting times due to high utilization of the operating 
RTGs in each block and congestion within the transport system of the yard. Hence it is clear that several criteria influence the performance of the operations at the yard.

The proposed fuzzy strategy provides decision support for Import-Dry containers with regards to minimizing relocation moves and distance traveled of the yard equipment by incorporating the real-time information for the aforementioned set of criteria of the yard and incoming containers.

\subsection{Phase I - Block Assignment}

Once a container enters the yard, the framework seeks to select a block that is most favorable with regards to a set of criteria. The following two criteria are selected for the study presented herein:

- Distance Block-Gate describes the distance from the landside entry point of a container (gate) to the yard, more precisely, the particular block considered.

- Block utilization is a measure that identifies the space used within a block. It is derived by taking the ratio of used allocation spaces $s_{i}^{\text {allocated }}$ and available allocation

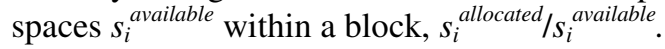

The corresponding output variable is the block VoG. The membership functions are of a triangular format for each input variable and output variable. Each variable has the shape as shown in the example in Figure 2 with Med representing the value at which the triangular membership function peaks. They are outlined in Table 1. The degree of membership $\mu$ of variable $x$ is determined as follows,

$$
\mu(x ; \text { Min,Med, Max })=\left\{\begin{array}{cl}
\frac{(x-\text { Min })}{(\text { Med }- \text { Min })} & \text { Min } \leq x \leq \text { Med } \\
\frac{(\text { Max }-x)}{(\text { Max }- \text { Med })} & \text { Med }<x \leq \text { Max } \\
0 & x<\text { Min } \\
0 & x>\text { Max }
\end{array}\right.
$$

Each membership function is associated with a linguistic term outlined as subset, which is presented in the second column of the table and may be small (or low), medium and high. The last three columns of the table determine each subset to be considered as shown in Eq.1, using minimum (min), medium (med) and maximum (max).

Particularly for import containers, the two input variables that are of interest correspond to the distance between the block and the gate and block utilization. Table 2 shows the corresponding set of rules for these input variables and the corresponding output variable block VoG. Each column of the table corresponds to each variable and the rows present the five rules considered herein based on the preferences of the managers of the yard at the port considered as case study. 
Table 1. Membership functions Phase I - block VoG

\begin{tabular}{|l|l|c|c|c|}
\hline \multicolumn{7}{|c|}{ Variable } & \multicolumn{1}{c|}{ Subset } & Min & Med & Max \\
\hline \multicolumn{5}{|c|}{ Input variables } \\
\hline Distance Block-Gate & Small & 0 & 0 & 50 \\
\hline Distance Block-Gate & Medium & 10 & 60 & 110 \\
\hline Distance Block-Gate & Large & 70 & 120 & 120 \\
\hline Block utilization & Low & 0 & 0 & 0.4 \\
\hline Block utilization & Medium & 0.1 & 0.5 & 0.9 \\
\hline Block utilization & High & 0.6 & 1 & 1 \\
\hline \multicolumn{5}{|c|}{ Output variables } \\
\hline block VoG & Low & 0 & 0 & 0.4 \\
\hline block VoG & Medium & 0.1 & 0.5 & 0.9 \\
\hline block VoG & High & 0.6 & 1 & 1 \\
\hline
\end{tabular}

Source: Self-elaborated based on the information of the case study.

Table 2. Extent of Phase I - block VoG rule base

\begin{tabular}{|l|l|l|l|}
\hline & $\begin{array}{l}\text { Distance } \\
\text { Block-Gate }\end{array}$ & $\begin{array}{l}\text { Block } \\
\text { utilization }\end{array}$ & $\begin{array}{l}\text { block } \\
\text { VoG }\end{array}$ \\
\hline 1 & Small & Low & High \\
\hline 2 & Small & High & Medium \\
\hline 3 & Medium & High & Small \\
\hline 4 & Medium & Medium & Small \\
\hline 5 & High & Medium & Small \\
\hline
\end{tabular}

(Source: Self-elaborated based on the information of the case study).

\subsection{Phase II - Stack Assignment}

Once a block has been selected, Phase 2 is initiated to determine the most preferable stack within the chosen block. Two criteria are being used to make this decision:

- Stack height enables to differentiate between low, medium and high stacks in the block.

- Estimated time of dispatching the container (ETD) on top of the particular stack is before, after or reasonably similar to the ETD of the container that is being allocated. This measure is related to the dwell time of the container, the permanence time of the container in the port. More precisely, the criterion considers the normalized difference between the ETD of the top container of the current stack and the ETD of the incoming container that has to be assigned with a position in the yard. This criterion takes a value between 0 and 1 with values larger than 0.5 indicating that the incoming container is estimated to be dispatched before the container on the top of the considered stack and values smaller than 0.5 , otherwise. 
The output variable is the stack VoG with a high value indicating a more favorable stack position. Membership functions are of triangular shape and determined as described in Eq. 1 with the particular characteristics shown in Table 3. The table presents each of the input and output variables, the corresponding subset (column 2) and the minimum, medium and maxim values (columns 3, 4 and 5 respectively). The corresponding set of rules to determine a stack VoG is presented in Table 4, where columns 2 and 3 correspond to the values of the input variables: Stack height and ETD. Column 4 presents the corresponding output variable stack VoG.

Table 3. Membership functions Phase II - stack VoG

\begin{tabular}{|c|c|c|c|c|}
\hline Variable & Subset & Min & Med & Max \\
\hline \multicolumn{5}{|c|}{ Input variables } \\
\hline Stack height & Low & 0 & 0 & 2 \\
\hline Stack height & Medium & 0 & 2 & 4 \\
\hline Stack height & High & 2 & 4 & 4 \\
\hline ETD & Earlier & 0 & 0 & 0.6 \\
\hline ETD & Later & 0.4 & 1 & 1 \\
\hline \multicolumn{5}{|c|}{ Output variables } \\
\hline stack $\mathrm{VoG}$ & Low & 0 & 0 & 0.4 \\
\hline stack $\mathrm{VoG}$ & Medium & 0.1 & 0.5 & 0.9 \\
\hline stack $\mathrm{VoG}$ & High & 0.6 & 1 & 1 \\
\hline
\end{tabular}

(Source: Self-elaborated based on the information of the case study).

Table 4. Extent of Phase II - stack VoG rule base

\begin{tabular}{|l|l|l|l|}
\hline \multicolumn{1}{|c|}{ Stack height } & \multicolumn{1}{c|}{ ETD } & \multicolumn{1}{c|}{ stack VoG } \\
\hline 1 & Low & Later & Small \\
\hline 2 & Medium & Later & Small \\
\hline 3 & Medium & Earlier & High \\
\hline 4 & High & Earlier & Medium \\
\hline
\end{tabular}

(Source: Self-elaborated based on the information of the case study).

\subsection{Relocation Phase I* Block VoG}

If a container is scheduled to be retrieved from the yard, relocation moves may be necessary in case the container is not accessible due to other containers stacked on top of it. The framework supports relocating containers based on phase I and II of the fuzzy algorithm. However, phase I which used to assign the most preferable block in the yard is amended in order to take into consideration the current position of a 'relocated' container in its current block. Therefore, the two criteria considered are:

- Distance block-block, distance between every block to the current block;

- Block utilization, space used within the current block; 
Table 5 presents the corresponding set of membership functions. For each variable, its subset is identified in column 2 and the minimum, medium and maximum values of each input variable and subset are presented in columns 3, 4 and 5. Table 6 presents the corresponding set of rule base. Column 2 and 3 present the values of the two input variables and column 4 the values of the output variable, block VoG.

Table 5. Membership functions - Phase I* Relocation block VoG

\begin{tabular}{|l|l|c|c|c|}
\hline Variable & Subset & Min & Med & Max \\
\hline \multicolumn{5}{|c|}{ Input variables } \\
\hline Distance Block-Block & Small & 0 & 0 & 50 \\
\hline Distance Block-Block & Medium & 10 & 60 & 110 \\
\hline Distance Block-Block & Large & 70 & 120 & 120 \\
\hline Block utilization & Low & 0 & 0 & 0.4 \\
\hline Block utilization & Medium & 0.1 & 0.5 & 0.9 \\
\hline Block utilization & High & 0.6 & 1 & 1 \\
\hline \multicolumn{7}{|c|}{ Output variables } \\
\hline block VoG & Low & 0 & 0 & 0.4 \\
\hline block VoG & Medium & 0 & 0.5 & 1 \\
\hline block VoG & High & 0.6 & 1 & 1 \\
\hline
\end{tabular}

(Source: Self-elaborated based on the information of the case study).

Table 5. Rule Base - Phase I* Relocation block VoG

\begin{tabular}{|l|c|c|c|c|c|c|c|}
\hline & $\begin{array}{c}\text { Distance } \\
\text { Block- } \\
\text { Block }\end{array}$ & $\begin{array}{c}\text { Block } \\
\text { utilization }\end{array}$ & $\begin{array}{c}\text { block } \\
\text { VoG }\end{array}$ & $\begin{array}{c}\text { Distance } \\
\text { Block- } \\
\text { Block }\end{array}$ & $\begin{array}{c}\text { Block } \\
\text { Utilization }\end{array}$ & $\begin{array}{c}\text { block } \\
\text { VoG }\end{array}$ \\
\hline 1 & Small & Low & High & 6 & Medium & High & Low \\
\hline 2 & Small & Medium & Medium & 7 & Large & Low & Medium \\
\hline 3 & Small & High & Low & 8 & Large & Medium & Low \\
\hline 4 & Medium & Low & Medium & 9 & Large & High & Low \\
\hline 5 & Medium & Medium & Low & & & & \\
\hline
\end{tabular}

(Source: Self-elaborated based on the information of the case study).

\section{$5 \quad$ Experimental Results}

This section reports the results of the computational testing of the proposed fuzzy logic framework. The performance of the procedure is evaluated with respect to other heuristic procedures taken from the literature as well as a random allocation procedure. The fuzzy logic framework was coded in Matlab, using the fuzzy logic toolbox. To test the procedure, instances are generated as described in Section 5.1 and three scenarios were tested. All testing was performed on an Intel Core i5 processor running at $1.6 \mathrm{GHz}$ with $4 \mathrm{~GB}$ of RAM. 


\subsection{Experimental Set-Up}

For computational experiments, we will apply the model proposed to the yard blocks of Import-Dry containers, considering that the container terminal has dedicated blocks for Import/Export containers as well as Reefer/Dry/IMO containers. Hence, blocks for Import and Dry containers are not shared with any other type of container. For the instances generated in order to test the performance of the model proposed herein, we will consider a port infrastructure in which the Import-Dry area contains five nonhomogeneous blocks. The number of bays, rows and tiers of each block is described in Table 7. Total static capacity accounts for 472 slots.

Table 6. Characteristics of the blocks of the instance

\begin{tabular}{|c|c|c|c|}
\hline Block & Bays & Rows & Tiers \\
\hline 1 & 5 & 6 & 4 \\
\hline 2 & 3 & 7 & 4 \\
\hline 3 & 5 & 6 & 4 \\
\hline 4 & 3 & 7 & 4 \\
\hline 5 & 2 & 8 & 4 \\
\hline
\end{tabular}

The entry point of the yard is considered as one berth from the quay side and the exit point is considered as the gate where external trucks arrive and depart from the terminal with the dispatched container (given that only Import-Dry containers are considered). Phase I rule base assumes a preference to locate import containers closer to the gate (in this case, import-dry). The impact of this criterion may vary depending on operational and infrastructural yard characteristics for structurally different terminal layouts. However, this preference is assumed to be of general interest for import containers as this aims to minimize internal truck congestion of the terminal. The layout of the Import-Dry yard of the container terminal is assumed to be as represented in Figure 3.

Three scenarios have been tested based on the initial inventory of containers at the yard and the arrival and departure rates of containers:

- Empty yard. This scenario assumes that at the beginning of the planning horizon the yard is empty, which is not realistic according to the real case, but useful to test the algorithmic behavior of the investigated stacking policies. Operational simulation: $\mathrm{T}=0$ to $\mathrm{T}_{\max }=337(\mathrm{~min})$, with containers arriving every minute and departing at a random point of time by $\mathrm{T}_{\max }$.

- Block utilization of $\mathbf{5 0 \%}$ in the yard. This scenario assumes that there exists an initial inventory of containers at the yard, based on a $50 \%$ of utilization, which can be considered as a non-congested period. Operational simulation: $\mathrm{T}=0$ to $\mathrm{T}_{\max }=$ $500(\mathrm{~min})$, with containers arriving every five minutes.

- Congested yard, block utilization of $\mathbf{8 0 \%}$ in the yard. This scenario assumes a congested yard with a high utilization. It is important to point out that the real cases are currently facing high congestion levels as container transfers is constantly 


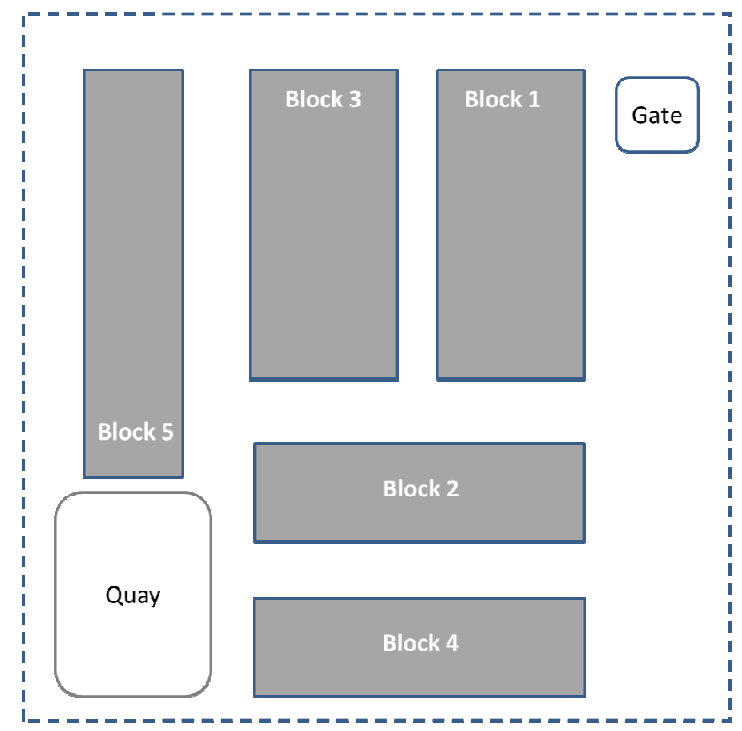

Fig. 3. Layout of the import-dry yard section

increasing while infrastructure has not been expanded. Operational simulation: $\mathrm{T}=0$ to $\mathrm{T}_{\max }=500(\mathrm{~min})$, with containers arriving every 5 minutes.

In order to evaluate the results of the computational testing, a relocation ratio is estimated, based on a comparison of the relocation moves with respect to total moves (effective + relocations) as it is shown in Eq. 2 .

$$
\text { Relocation ratio } r=\frac{\text { Relocation moves }}{\text { Relocation moves }+ \text { effective moves }}
$$

Furthermore, the distance of moving the container from its position in the yard to the exit (gate) is evaluated - including any required relocation distance. This will allow for investigating the impact of integrated distance criteria and provides an indication for a potential risk of congestion and inefficiency associated with the applied stacking policy.

\subsection{Stacking Policies Evaluated}

The following policies have been investigated to understand the impact and level of competitiveness of the proposed Fuzzy Logic framework:

- RAND: A container is assigned to a random position within the yard without taking into consideration any preference related to the position.

- RSDT: This policy has been introduced by [3] but has been amended into a 2-Phase strategy such that a block is randomly selected under the consideration that at least one stack includes a top container that has an ETD later than the incoming container 
that requires stacking. A stack is then chosen randomly among stacks with previous characteristics. If there is no stack for which this is the case, then a stack is randomly chosen within the yard.

- Levelling: An incoming container is assigned to the lowest stack in the yard which means filling up all empty stacks first and then step by step levelling the height of stacks within the yard.

- LDT (Less difference time): Find the stack for which the difference in ETD of the container on the top and the ETD of the incoming container is minimized. If no stack like that exists, assign the incoming container to an empty stack. If there is no empty stack, assign to the highest stack. If there are several stacks with the same maximum height, then take the one closest to the transfer point (which given that we consider only import-dry containers, is the gate) [3].

- Fuzzy Logic Framework: Multi-criteria framework proposed herein and described with detail in Section 4.

From the above policies, the port terminal considered as a case study applies a hybrid policy between RAND and Levelling policies described before. This is due to the crane operator assigning the location of the containers based on his experience with the only aim of avoiding blocking space that cannot be further utilized so the priority is to fill up stacks. However, this is very variable and dependent on the crane operator so it can be considered similar to a random policy.

Each algorithm has been run 10 times (replicates) using a random set of arriving containers. The averages and standard deviation of the relocation ratio and the travel distance are reported in Tables 8 and 9, respectively. Each table presents the results obtained for each algorithm and each of the three scenarios tested. Columns 2 and 3 present the results for the empty yard scenario, with the average and standard deviation values respectively. Columns 4 and 5 for the scenario with $50 \%$ of congestion and columns 6 and 7 for the scenario with $80 \%$ of congestion, for the relocation ratio and the travel distance in each table respectively. Rows correspond to each of the algorithms tested.

Table 7. Results by performance metric: Relocation ratio

\begin{tabular}{|l|c|c|c|c|c|c|}
\hline Algorithm & $\begin{array}{c}\text { Relocation } \\
\text { ratio }\end{array}$ & $\begin{array}{c}\text { Relocation } \\
\text { ratio } \\
\text { (Stdev) }\end{array}$ & $\begin{array}{c}\text { Relocation } \\
\text { ratio }\end{array}$ & $\begin{array}{c}\text { Relocation } \\
\text { ratio } \\
\text { (Stdev })\end{array}$ & $\begin{array}{c}\text { Relocation } \\
\text { ratio }\end{array}$ & $\begin{array}{c}\text { Relocation } \\
\text { ratio } \\
\text { (Stdev) }\end{array}$ \\
\hline & \multicolumn{2}{|c|}{ Empty yard } & \multicolumn{2}{|c|}{$\mathbf{5 0 \%}$ congestion } & \multicolumn{2}{|c|}{$\mathbf{8 0 \%}$ congestion } \\
\hline Random & 0.080 & 0.010 & 0.190 & 0.020 & 0.270 & 0.010 \\
\hline RSDT & 0.110 & 0.020 & 0.250 & 0.020 & 0.330 & 0.010 \\
\hline Levelling & 0.000 & 0.000 & 0.090 & 0.010 & 0.190 & 0.010 \\
\hline LDT & 0.070 & 0.010 & 0.040 & 0.003 & 0.050 & 0.004 \\
\hline Fuzzy & 0.000 & 0.000 & 0.070 & 0.003 & 0.180 & 0.004 \\
\hline
\end{tabular}


Table 8. Results by performance metric: Distance travelled

\begin{tabular}{|c|c|c|c|c|c|c|}
\hline Approach & Distance & $\begin{array}{c}\text { Distance } \\
\text { (Stdev) }\end{array}$ & Distance & $\begin{array}{c}\text { Distance } \\
\text { (Stdev) }\end{array}$ & Distance & $\begin{array}{c}\text { Distance } \\
\text { (Stdev) }\end{array}$ \\
\hline & \multicolumn{2}{|c|}{ Empty yard } & \multicolumn{2}{|c|}{$50 \%$ congestion } & \multicolumn{2}{|c|}{$80 \%$ congestion } \\
\hline Random & $29,616.000$ & $1,103.910$ & $36,195.300$ & $1,307.660$ & $57,808.600$ & 897.060 \\
\hline RSDT & $30,313 \cdot 100$ & $2,973.370$ & $32,586.800$ & $2,931.340$ & $55,087.500$ & $5,446.130$ \\
\hline Levelling & $24,258.500$ & 601.980 & $27,959.700$ & 537.770 & $43,318.100$ & 707.970 \\
\hline LDT & $33,000.900$ & 839.940 & $32,953.100$ & 628.750 & $46,591.400$ & 415.260 \\
\hline Fuzzy & $24,262.100$ & 500.460 & $26,323.400$ & 824.040 & $46,740.300$ & 178.470 \\
\hline
\end{tabular}

As it can be observed in the tables, the obtained results highly depend on the scenario tested and the performance metric considered. For an empty yard scenario, the fuzzy algorithm outperforms the rest of the algorithms both for the distance and relocation metrics on average while also showing least variability by low standard deviations. For a $50 \%$ of congestion scenario, the fuzzy algorithm outperforms the average distance and relocation ratio with respect to the rest of algorithms, but the levelling algorithm presents a lower value of the standard deviation for the distance metric indicating a more consistent performance. For a congested scenario ( $80 \%$ utilization), the LDT heuristic presents a better performance on the relocations metric, followed by the fuzzy algorithm with both presenting the same standard deviation value. For the distance metric, the levelling algorithm presents a better average value, but the fuzzy algorithm shows less variability in its performance. It is worthy to observe that for the distance metric, the random and RSDT algorithms present higher values of the standard deviation, while the LDT and fuzzy algorithms are more consistent.

As observed in the results, a random assignment does not perform well compared to other strategies, so it is clear that there is need for improvements at those ports that do not have mechanism to support the online assignment of containers to the yard and rely on the crane operator to take this decision based on his experience.

Hence, we can observe in general a good performance of the fuzzy algorithm proposed with respect to other algorithms. Although the fuzzy algorithm does not outperform the rest of the heuristics for all the scenarios evaluated, it is a new strategy to include a set of different criteria and potential infrastructural characteristics of the yard and containers based on different performance metrics. This approach has the advantage of dealing with a reasoning that is approximate rather than fixed and exact which is useful for situations where it is difficult to define a priori if certain criteria are more relevant with respect to another.

\section{Conclusions and Recommendations for Further Research}

The presented approach uses a 2-Phase framework based on a fuzzy rule concept to support the decision of assigning space to incoming containers at the yard of a container terminal. The approach is well suited for situations where imprecise information and a high degree of uncertainty are experienced. The presented fuzzy structure 
enables real-time decision making in the form of assigning any incoming container to the most preferable location in the yard. The system is highly flexible and adjustable to different infrastructures and operational preferences with regards to container traffic through the yard.

The experimentation is planned to be extended to a set of case studies to investigate whether there exist particular differences in operational preferences or whether a generic preference may be applied on common port infrastructures. Moreover, the system is currently considering only a selection of criteria (e.g. block-specific and container-specific) and performance measures (rate of relocation moves and distance traveled) which may benefit from an extension so that other aspects can be considered such as congestion within the yard.

Experimental results show a good performance of the algorithm with respect to other algorithms proposed in the literature and a random assignment. Although the fuzzy algorithm does not outperform all the algorithms for all the scenarios tested and metrics evaluated, it shows a good performance with low variability of the results obtained. This indicates that the fuzzy logic approach is a good strategy for the container stacking problem that has not been employed previously and has the advantage of dealing with a reasoning that is approximate rather than fixed and exact. This is useful for situations where it is difficult to define a priori if a certain metric is more relevant with respect to another, or when it is hard to provide a precise evaluation of a solution with respect to several criteria.

As further research, we may explore how flexible it is to add criteria in the fuzzy logic framework with respect to other approaches for the container stacking problem found in the literature. It is also envisaged to integrate this framework with a monitoring strategy that allows understanding the risk and reasons of relocation in the port under the assumption of no disruptive event. This would further enable the framework to indicate at which point a potential reorganization of the yard (or particular areas of the yard) is required.

Acknowledgments. The authors would like to thank Stefan Voß and Dario Pacino for several useful comments and suggestions. Addressing the issues raised has allowed us to greatly improve the paper.

\section{References}

1. Bazzazi, M., Safaei, N., Javadian, N.: A genetic algorithm to solve the storage space allocation problem in a container terminal. Computers \& Industrial Engineering 56(1), 44-52 (2009)

2. Bierwirth, C., Meisel, F.: A survey of berth allocation and quay crane scheduling problems in container terminals. European Journal of Operational Research 202(3), 615-627 (2010)

3. Borgman, B., van Asperen, E., Dekker, R.: Online rules for container stacking. OR Spectrum 32(3), 687-716 (2010)

4. Bortfeldt, A., Forster, F.: A tree search procedure for the container pre-marshalling problem. European Journal of Operational Research 217(3), 531-540 (2012)

5. Carlo, H.J., Vis, I.F.A., Roodbergen, K.J.: Storage yard operations in container terminals: literature overview, trends, and research directions. European Journal of Operational Research 235(2), 412-430 (2014) 
6. Caserta, M., Voß, S.: Sniedovich:Applying the corridor method to a blocks relocation problem. OR Spectrum 33(4), 915-929 (2011)

7. Caserta, M., Schwarze, S., Voß, S.: A mathematical formulation and complexity considerations for the blocks relocation problem. European Journal of Operational Research 219(1), 96-104 (2012)

8. Castilho, B.D., Daganzo, C.F.: Handling strategies for import containers at marine terminal. Transportation Research Part B 27(2), 151-166 (1993)

9. Chen, L., Lu, Z.: The storage location assignment problem for outbound containers in a maritime terminal. International Journal of Production Economics 135(1), 73-80 (2012)

10. Dekker, R., Voogd, P., van Asperen, E.: Advanced methods for container stacking. OR Spectrum 28(4), 563-586 (2006)

11. Expósito-Izquierdo, C., Melián-Batista, B., Moreno-Vega, M.: Pre-marshalling problem: Heuristic solution method and instances generator. Expert Systems with Applications 39(9), 8337-8349 (2012)

12. Forster, F., Bortfeldt, A.: A tree search procedure for the container relocation problem. Computers \& Operations Research 39(2), 299-309 (2012)

13. Gharehgozli, A.H., Yu, Y., de Koster, R., Udding, J.T.: A decision-tree stacking heuristic minimising the expected number of reshuffles at a container terminal. International Journal of Production Research 52(9), 2592-2611 (2014)

14. Huang, S.-H., Lin, T.-H.: Heuristic algorithms for container pre-marshalling problems. Computers \& Industrial Engineering 62(1), 13-20 (2012)

15. Kang, J., Ryu, K.R., Kim, K.H.: Deriving stacking strategies for export containers with uncertain weight information. Journal of Intelligent Manufacturing 17(4), 399-410 (2006)

16. Kim, K.H.: Evaluation of the number of re-handles in container yards. Computers \& Industrial Engineering 32(4), 701-711 (1997)

17. Kim, K.H., Hong, G.-P.: A heuristic rule for relocating blocks. Computers \& Operations Research 33(4), 940-954 (2006)

18. Kim, K.H., Kim, H.B.: Segregating space allocation models for container inventories in port container terminals. International Journal of Production Economics 59(1), 415-423 (1999)

19. Kim, K.Y., Kim, K.H.: A routing algorithm for a single straddle carrier to load export containers onto a containership. International Journal of Production Economics 59(1), 425433 (1999)

20. Kim, K.H., Lee, K.M., Hwang, H.: Sequencing delivery and receiving operations for yard cranes in port container terminals. International Journal of Production Economics 84(3), 283-292 (2003)

21. Kim, K.H., Park, Y.M., Ryu, K.R.: Deriving decision rules to locate export containers in container yard. European Journal of Operational Research 124(1), 89-101 (2000)

22. Kim, K.H., Park, K.T.: A note on a dynamic space-allocation method for outbound containers. European Journal of Operational Research 148(1), 92-101 (2003)

23. Kim, K.H., Park, Y.-M., Jin, M.-J.: An optimal layout of container yards. OR Spectrum 30(4), 675-695 (2008)

24. Kozan, E.: Optimizing container transfers at multimodal terminals. Mathematical and Computer Modelling 31(10-12), 235-243 (2000)

25. Lan, L.W., Kao, C.-K.: A comparison of stacking efficiency for various strategies of slot assignment in container yards. Journal of the Eastern Asia Society for Transportation Studies 4(1), 300-318 (2001) 
26. Lee, D.H., Cao, Z., Meng, Q.: Scheduling of two-transtainer systems for loading outbound containers in port container terminals with simulated annealing algorithm. International Journal of Production Economics 107(1), 115-124 (2007)

27. Lee, B.K., Kim, K.H.: Optimizing the block size in container yards. Transportation Research Part E: Logistics and Transportation Review 46(1), 120-135 (2010)

28. Lim, A., Xu, Z.: A critical-shaking neighborhood search for the yard allocation problem. European Journal of Operational Research 174(2), 1247-1259 (2006)

29. Park, R.C.T., Kim, Y.H., Ryu, K.R.: Dynamic adjustment of container stacking policy in an automated container terminal. International Journal of Production Economics 133(1), 385-392 (2011)

30. Petering, M.E.H.: Real-time container storage location assignment at an RTG-based seaport container transshipment terminal: problem description, control system, simulation model, and penalty scheme experimentation. Flexible Services and Manufacturing Journal, 1-31 (2013)

31. Rodrigue, J.P., Notteboom, T.: The terminalization of supply chains: reassessing the role of terminals in port / hinterland logistical relationships. Maritime Policy and Management 36(2), 165-183 (2009)

32. Saurí, S., Martín, E.: Space allocating strategies for improving import yard performance at marine terminals. Transportation Research Part E: Logistics and Transportation Review 47(6), 1038-1057 (2011)

33. Sculli, D., Hui, C.F.: Three dimensional stacking of containers. OMEGA 16(6), 585-594 (1988)

34. Sniedovich, M., Voß, S.: The corridor method: a dynamic programming inspired metaheuristic. Control and Cybernetics 35(3), 551-578 (2006)

35. Stahlbock, R., Voß, S.: Operations Research at Container Terminals: A literature update. OR Spectrum 30(1), 1-52 (2008)

36. Steenken, D., Voß, S., Stahlbock, R.: Container terminal operations and operations research- a classification and literature review. OR Spectrum 26(1), 3-49 (2004)

37. Taleb-Ibrahimi, M., Castilho, B., Daganzo, C.F.: Storage space vs handling work in container terminals. Transportation Research Part B 27(1), 13-32 (1993)

38. Watanabe, I.: Characteristics and analysis method of efficiencies of container terminal - an approach to the optimal loading/unloading method. Container Age, 36-47 (1991)

39. Woo, Y.J., Kim, K.H.: Estimating the space requirement of outboundcontainer inventories in port container terminals. International Journal of Production Economics 133(1), 293$301(2011)$

40. van Asperen, E., Borgman, B., Dekker, R.: Evaluating impact of truck announcements on container stacking efficiency. Flexible Services and Manufacturing Journal 25(4), 543-556 (2012)

41. Zadeh, L.A.: Fuzzy Sets. Information and Control 8(3), 338-353 (1965)

42. Zhang, C., Liu, J., Wan, Y.W., Murty, K.G., Linn, R.J.: Storage space allocation in container terminals. Transportation Research Part B 37(10), 883-903 (2003)

43. Zhao, W., Goodchild, A.: The impact of truck arrival information on container terminal rehandling. Transportation Research Part E Logistics and Transportation Review 46(3), 327-343 (2010) 